\title{
application combinée de l'analyse structurale, de la géostatistique et de la théorie des blocs clés à l'étude des problèmes de stabilité et de confortement des travaux miniers
}

\author{
combined application of structural analysis, geostatistics \\ and key-block theory in the study of problems of stability \\ and reinforcement on mining works
}

\author{
M. VINCHES \\ Ecole des Mines d'Alès* \\ Bureau de Recherches Géologiques et Minières**
}

Rev. Franç. Géotech. n` 45, p.p. $59-70$ (octobre 1988)

\section{Résumé}

L'étude présentée ici vise la mise en place d'une méthodologie qui permette le dimensionnement d'ouvrages miniers, souterrains ou non, en fonction de la structure géologique du massif rocheux dans lequel ils se trouvent.

Trois étapes de cette méthodologie sont exposées sucessivement:

- la détermination des caractéristiques statistiques et géostatistiques des populations de fractures :

- la génération, par simulation, d'un champ de fractures de caractéristiques statistiques données:

- I'étude de la stabilité de blocs isolés, définis par ces fractures ; ceci grâce à la théorie des blocs clés.

A la suite de cette chaine de traitement des données structurales, des règles d'action devront être tirées, concernant la conception et l'exécution des ouvrages à réaliser.

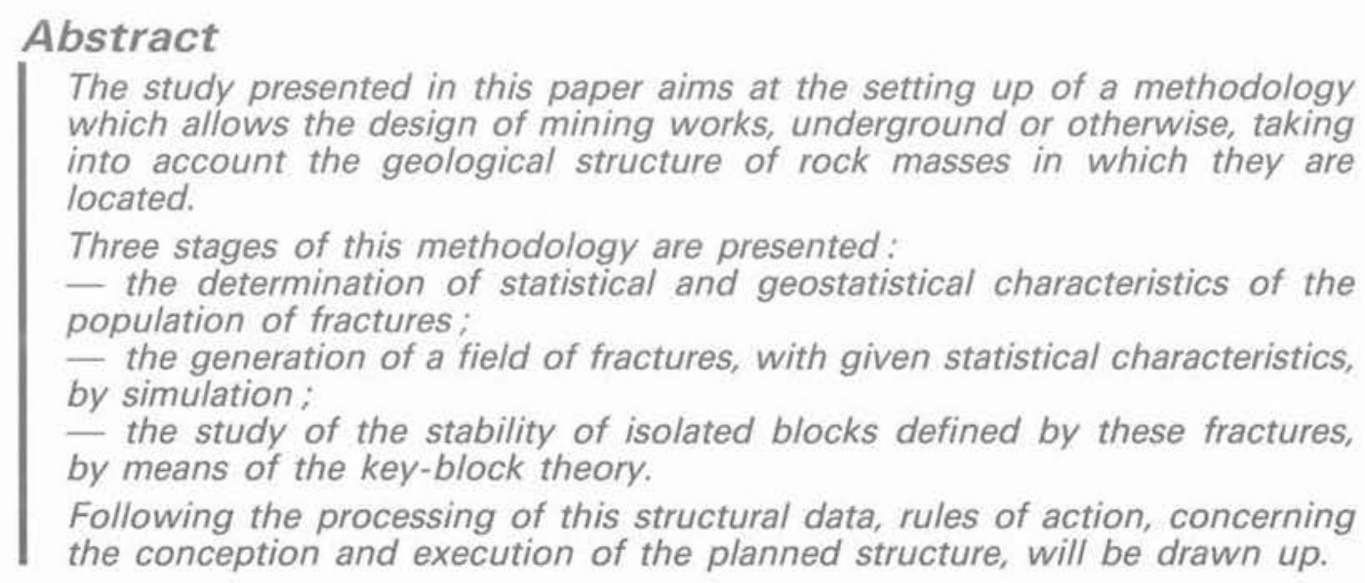

- 6 avenue de Clavières, 30107 Alès Cedex.

* Département d'ingénierie géotechnique, BP 6009, 45060 Orléans Cedex 02. 


\section{INTRODUCTION}

Dans le cadre d'une modélisation et d'une étude de stabilité de massifs rocheux fracturés, considérés comme assemblages de blocs, on peut généralement distinguer deux étapes qui sont successivement, la détermination de la géométrie des blocs liée à la géologie du site, et l'étude de la stabilité des dits blocs. Plusieurs types d'approches, statistiques ou déterministes, peuvent être envisagés pour la caractérisation de la géométrie des blocs (D. HELIOT, 1987 ; J. FINE, T. KORINI, 1987 ; M. VINCHES, 1987; P.M. WARBURTON, 1985), comme pour l'étude de leur stabilité (P. CUNDALL, 1980; P. CUNDALL et al., 1985; J.LEMOS et al., 1987 ; L.J. LORIG et al., 1986; R. GOODMAN et G.H. SHI, 1985 ; P.M. WARBURTON, 1987 ; J.FINE et al., 1987).

La démarche suivie dans cette étude se base sur les travaux en géologie structurale de H. MASSOUD (1987); H. MASSOUD et J.P. CHILES (1986); J.P. CHILES (1987), permettant la modélisation statistique et géostatistique d'un champs de fractures. On présente le cas d'études réalisées sur deux mines françaises: la mine souterraine de Fanay-Augeres de la COGEMA, la mine à ciel ouvert des Bondons de la Compagnie Française de MOKTA.

Dans une deuxième partie, on expose comment grâce à des programmes de génération de champs de fractures (H.M. Peggy GILMOUR et al., 1986; D. BILLAUX, 1986) il est possible d'obtenir une réalisation particulière du phénomène aléatoire, étudié dans la première partie, de paramètres statistiques connus.

On décrit ensuite comment la connaissance des intersections des fractures engendrées doit pouvoir permettre la détermination de blocs, délimités par les plans de fractures, de dimensions finies. On pourra alors étudier leur stabilité, grâce à la théorie des blocs clés, et en déduire des règles d'action concernant la conception (dimensionnement, orientation, forme,...) d'ouvrages miniers.

En conclusion sont présentés quelques points de discussion, et l'état actuel des recherches menées sur ce sujet à l'Ecole des Mines d'Alès, et au B.R.G.M.

Il convient de noter que la méthodologie exposée en est encore à un stade de recherche, et n'a été appliquée, à ce jour, que sur des sites expérimentaux dans le cas de schistes et de granites.

\section{MODÉLISATION DU CHAMP DE FRACTURES - CAS D'APPLICATION}

Cette partie a pour objet de préciser les caractéristiques spécifiques de la modélisation des champs de fractures par les techniques de la géostatistique, par rapport aux techniques basées sur la «statistique classique», ces dernières ne permettant pas de prendre en compte la structuration spatiale des phénomènes naturels étudiés. Nous basant sur les travaux réalisés par H. MASSOUD et J.P. CHILES principalement, nous reprendrons la démarche complète permettant d'aboutir à ce type de modélisation, en trois étapes principales: l'obtention des données de base, suivie de l'analyse structurale, puis de la modélisation, de la fracturation. Nous ferons appel à des résultats obtenus sur deux sites diffërents: la mine souterraine de Fanay-Augeres (Haute-Vienne) dans le granite du massif de Saint-Sylvestre, et la mine à ciel ouvert des Bondons (Lozère), principalement dans des schistes métamorphiques et quartzites de la série cévenole, au contact du batholite du Mont-Lozère.

\subsection{Obtention des données de base}

Nous distinguerons dans cette partie, les relevés de fractures, du traitement statistique qui en est fait, avant de faire part de quelques remarques de portée générale.

\section{Les relevés de fractures}

Les relevés ont été effectués, selon les trois phases suivantes:

1. lavage de la partie de galerie ou d'affleurement étudiée ;

2. carroyage à la peinture ;

3. numérotation et repérage des fractures.

Ainsi, d'une part, toutes les fractures sont mises en évidence, d'autre part elles peuvent être aisément re. pérées par rapport à un référentiel global pour l'en. semble de la station de mesure. Enfin la dernière étape permet le comptage des fractures en s'assurant de les prendre toutes en compte.

Au total, ce sont 2940 fractures qui ont été ainsi échantillonnées, en galeries pour la mine Fanay, 2317 fractures en affleurements pour la mine des Bondons.

\section{Le traitement statistique}

Afin de caractériser chaque fracture, divers paramètres ont été sélectionnés. A une analyse statistique globale de l'ensemble des fractures dont le dépouillement se serait sans doute révélé difficile quant à l'interprétation des résultats, deux types de démarches pouvaient être préférés :

1. constituer des familles de fractures regroupées d'après leur origine tectonique, ou leur histoire ;

2. constituer des familles de façon subjective, à partir de stéréogrammes sur canevas de WULFF.

En pratique, dans le cas de Fanay, étudié antérieurement au site des Bondons, sur quelques 200 fractures, H. MASSOUD a cherché par une étude préalable, s'il y avait corrélation entre les démarches 1 et 2 . L'inconvénient de la première approche est de multiplier les familles, et de perdre l'information concernant les fractures non prises en compte dans les limites de définition des familles tectoniques principales. La deuxième méthode - distribution des fractures en familles dépendant du choix de l'opérateur -, engloble la distribution précédente; elle permet de conserver $80 \%$ des fractures levées dans une station donnée. En outre cette 
méthode a l'avantage d'une certaine souplesse d'utilisation, permettant de faire intervenir l'expérience de géologues de terrain ayant une bonne connaissance du site.

Une troisième démarche pour la constitution de familles, visant à prendre en compte la totalité des relevés de fractures consiste à générer les frontières de familles autour de pics de fréquence jusqu'à la rencontre des frontières des autres familles. L'inconvénient majeur de cette dernière façon de procéder, est d'englober dans une même famille, des fractures d'origine tectonique différente.

Pour le cas des Bondons, en revanche, la totalité des paramètres caractéristiques d'une fracture ont été relevés, permettant une étude structurale plus fine, servant d'autres buts que la seule étude de la stabilité de blocs rocheux. Il s'agit, notamment, de la mise en évidence de directions de schistosité variant selon les stations de mesures, et de corrélations entre directions, de structures particulières et la présence de divers matériaux de remplissage (M. VINCHES et al., 1988).

\section{Remarques générales}

Les familles directionnelles perpendiculaires aux plans d'observation sont mieux estimées que celles qui leur sont parallèles. Sauf dans le cas de sondages verticaux, les familles horizontales sont très mal représentées. Dans une note technique, J.L. YOW Jr (1987), donne des indications sur les problèmes de «zones aveugles », sur les pondérations à mettre en place pour tenir compte des biais introduits lors des mesures, et sur la prise en compte d'erreurs de mesures.
On note à ce sujet que si les stations de mesure sont disposées de façon aléatoire, leur direction liée à une disposition des supports constitués par des parements de galeries (cas de Fanay), ou un gradin à ciel-ouvert (cas des Bondons), est par contre imposé.

L'étude statistique du lot de fractures ainsi recueillies et traitées confirme l'organisation des fractures en familles, connues des géologues, dont on essaiera d'étudier la structure spatiale.

\subsection{Analyse structurale}

L'analyse faite dans le cas de Fanay-Augeres est basée sur des supports rectangulaires de taille constante de 5 mètres de long par 2 mètres de haut. Dans un cas autre (cas des Bondons par exemple), il faudra de même définir une taille de support dans lequel seront décrites les variables. Il convient lors de ce choix, de se rappeler que seules les structures de portée supérieure à la taille du support seront éventuellement révélées par l'analyse structurale.

Les variables qui ont été étudiées peuvent être considérées comme appartenant à deux types distincts :

- variables ordinaires visant à rendre compte de la structuration des fractures en terme de nombre de centres et de longueur cumulée, de façon à estimer et simuler des densités de fractures:

- variables particulières telles que la distance interfracturale horizontale qui, pour les farnilles sub-verticales, a été étudiée avec deux variantes: distance interfracturale en nombre et en mesure, ceci essentiellement pour contrôler si la distribution spatiale des

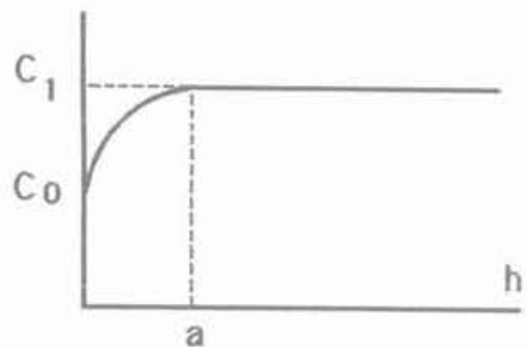

\begin{tabular}{|c|c|c|c|c|}
\hline \multirow{2}{*}{$\begin{array}{l}\text { ERREURS } \\
\text { DE } \\
\text { MESURES }\end{array}$} & \multirow[b]{2}{*}{ FAMILLES } & \multirow[b]{2}{*}{ PARAMETRES } & \multicolumn{2}{|c|}{ STATION: LASSAGNE } \\
\hline & & & DIRECTION & PENDAGE \\
\hline \multirow{5}{*}{ 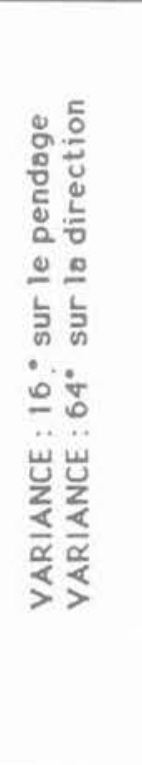 } & 1 & $\begin{array}{l}C_{0} \\
C_{1} \\
a\end{array}$ & $\begin{array}{r}1000 \\
1250 \\
6\end{array}$ & $\begin{array}{r}120 \\
300 \\
6\end{array}$ \\
\hline & 2 & $\begin{array}{l}c_{0} \\
c_{1} \\
0\end{array}$ & $\begin{array}{r}200 \\
250 \\
6 \\
\end{array}$ & $\begin{array}{r}38 \\
50 \\
6 \\
\end{array}$ \\
\hline & 3 & $\begin{array}{l}c_{0} \\
c_{1} \\
a\end{array}$ & $\begin{array}{r}130 \\
315 \\
12\end{array}$ & $\begin{array}{c}60 \\
60 \\
\infty\end{array}$ \\
\hline & 4 & $\begin{array}{l}C_{0} \\
C_{1} \\
a\end{array}$ & $\begin{array}{r}210 \\
320 \\
5 \\
\end{array}$ & $\begin{array}{r}44 \\
53 \\
5 \\
\end{array}$ \\
\hline & 5 & $\begin{array}{l}c_{0} \\
c_{1} \\
0\end{array}$ & $\begin{array}{r}170 \\
250 \\
5\end{array}$ & $\begin{array}{r}53 \\
95 \\
5\end{array}$ \\
\hline
\end{tabular}

Fig. 1 a. - Variogrammes sur les orientations, d'après H.MASSOUD - 1987.
Fig. 1 a - Variogrammes on the orientations. from H. MASSOUD - 1987. 
fractures est aléatoire. La variable distance interfracturale en mesure est définie en tout point d'une ligne horizontale $\mathrm{h}$ du parement. Elle prend comme valeur l'espacement existant entre la première fracture à gauche et la première fracture à droite de ce point, intersectant cette ligne h. Pour la variable distance interfracturale en nombre, on affecte un numéro à chaque intersection d'une fracture avec la ligne horizontale h, et une valeur de distance interfracturale.

Ces variables étant définies, il a été procédé, dans le cas de Fanay, à une analyse variographique sur plusieurs d'entre elles, dont les résultats ont donné lieu aux interprétations physiques suivantes, éventuellement transposables à d'autres cas d'observations:

- Effet de pépite :

sur les variogrammes estimés sur les variables caractérisant l'orientation d'un plan de fracture, la discontinuité à l'origine du variogramme (Fig. 1a) signifierait, selon H. MASSOUD et al (1986), que deux fractures proches, dans une même famille, ne sont pas rigoureusement parallèles, et peuvent donc se croiser. Géologiquement ce phénomène se conçoit bien, et semble lié à la définition des frontières des familles. $\mathrm{Si}$ la variabilité de l'orientation entre en jeu (la dispersion est visible sur le canevas de WULFF), l'erreur de mesure, dont nous avons déjà noté l'importance, intervient également dans ce phénomène.

\section{- Composante continue :}

cette composante est caractérisée par un palier et une portée. Dans le cas étudié (distance interfracturale en mesure), deux structures gigognes ont été mises en évidence, de portées distinctes vers 5-6 mètres, et environ 30 mètres. La première pourrait correspondre à un regroupement des fractures en paquets de longueur égale à 5.6 mètres. La deuxième serait la distance séparant ces paquets de fractures, situés à environ 30 mètres les uns des autres.

Un autre aspect important de l'étude de H. MASSOUD a été la mise en évidence d'un seuil de sélection des fractures, appelé seuil critique. Dans le but de rendre possible, en pratique, la définition de la structuration de la fracturation aux grandes distances, il était nécessaire d'avoir des relevés sur des stations de longueur importante, de l'ordre de la centaine de mètres. Afin de gagner du temps pour les opérations de relevés sur le terrain, mais avec le souci de conserver une information suffisante sur la structuration de la fracturation, diverses études ont été réalisées à partir des variogrammes, afin de déterminer si on pouvait se passer d'effectuer certains relevés, sans perdre d'information de façon significative. Ainsi dans le cas de FanayAugeres, a-t-on pu mettre en évidence un seuil critique pour la variable "distance interfracturale " égal à 1,5 mètre. La figure 16 montre, en effet, qu'il n'y a pas de changement de lallure du variogramme pour des longueurs de trace inférieures à 1,5 mètre.

En conclusion, et d'un point de vue global, il est préconisé une démarche en trois temps, permettant d'appréhender la variabilité de la fracturation aux moyennes et grandes distances:

1. Un levé systématique des fractures de longueur supérieure à $0,20 \mathrm{~m}$, les fractures de taille inférieure définissant des blocs de taille assez faible pour être dégagés lors du purgeage par exemple, sur des stations de mesure d'une longueur de lordre d'une centaine de mètres;

2. La détermination d'un seuil de sélection sur la longueur des traces des fractures (seuil critique);

3. Le levé d'autres stations de mesures en se limitant aux fractures dont la longueur de trace est supérieure au seuil de sélection critique.

Ce n'est que sur l'ensemble des données obtenues que sera calé un modèle probabiliste, rendant compte de la structuration du champ de fractures étudié.

\subsection{Modélisation de la petite fracturation}

La mise en cuure de cette modélisation peut se décomposer en trois temps :

1. Choix d'un type de modèle;

2. Mise en place d'une méthode d'estimation des paramètres du modèle;

3. Test sur la bonne adéquation du modèle à la réalité expérimentale.

Il nous a paru important de détailler cette démarche sur l'exemple de Fanay, à titre d'illustration.

Trois modèles successifs ont été considérés :

\section{Phénomène poissonien}

La confrontation des variogrammes expérimentaux aux résultats théoriques d'un phénomène poissonien conduit au rejet de ce modèle. En effet, si le variogramme sur les distances interfracturales en nombre semble présenter une allure pépitique pure correspondant à un phénomène poissonien, les variogrammes sur les distances interfracturales en mesure d'une part, sur le nombre de centres de fractures de l'autre, présentent une allure qui infirme l'hypothèse poissonienne à densité constante.

\section{Processus des salves aléatoires}

Ce type de processus est caractérisé de la façon suivante:

- les germes poissoniens sont implantés avec une densité $\varnothing \mathrm{g}$;

- le nombre de points dans un nuage centré en $\mathrm{x}$, est $\mathrm{N}(\mathrm{x})$;

- les nombres de point par nuage sont indépendants entre eux :

- chaque point du nuage est implanté en $\mathrm{X}+\mathrm{U}$ par rapport au germe qui est implanté en X, indépendamment des autres points selon une loi de probabilité $F(X)$. (On a le choix entre différentes fonctions de répartition (exponentielle, gaussienne, uniforme),... la fonction gaussienne a été retenue, a priori, par les auteurs H. MASSOUD et J.P. CHILES.)

En étudiant les schémas permettant de visualiser la disposition spatiale des traces de fractures à deux dimensions, les fractures apparaissent nettement regroupées en paquets, ou nuages, de quelques unités très 


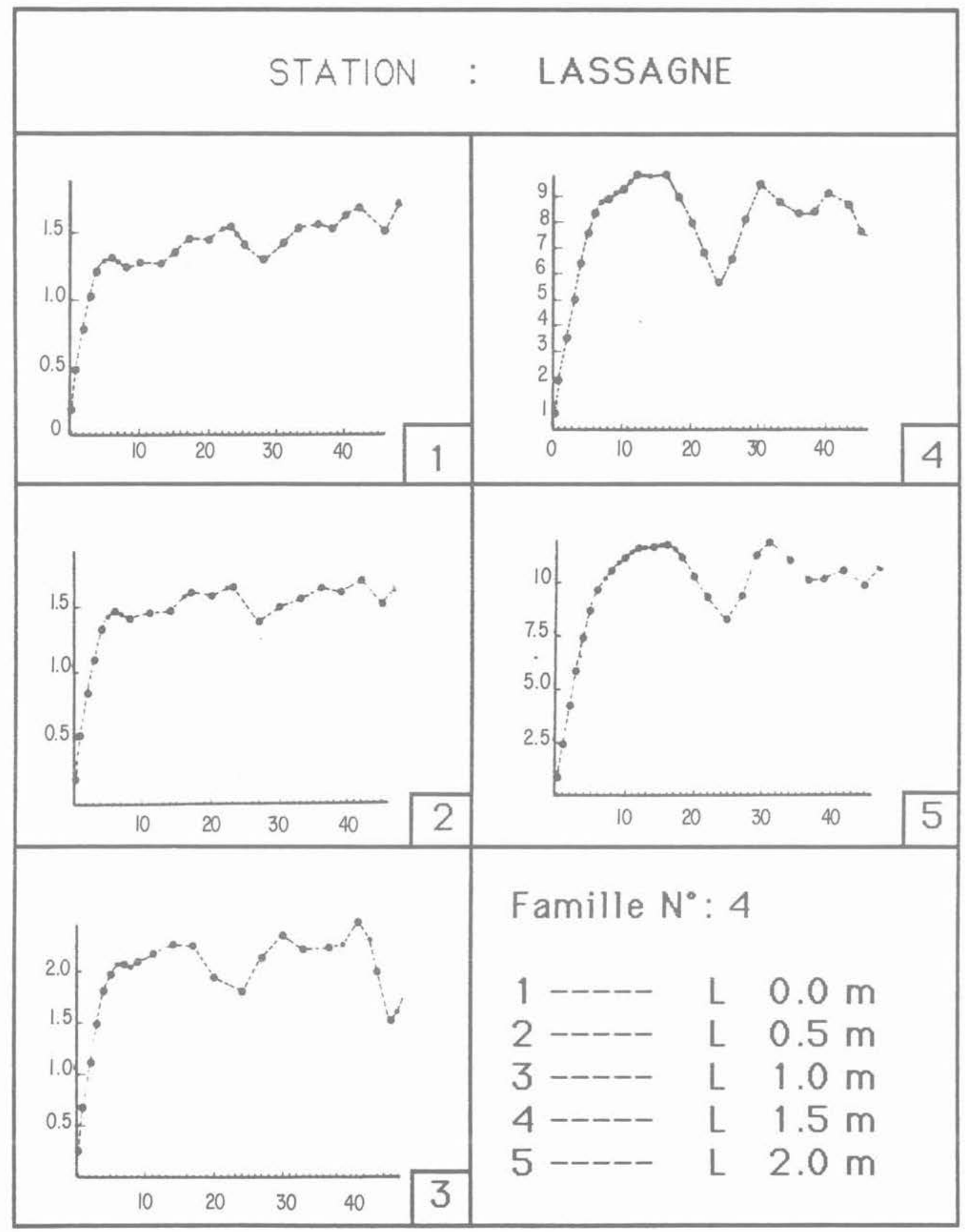

Fig. 1 b. - Variogrammes sur les distances interfracturales en mesure, d'après H. MASSOUD - 1987.

Fig. 1 b. - Variogrammes on the measurements of spacing of fractures, from H. MASSOUD - 1987. 
STATION : LOISEAU 320 ---> FAMILLE 4

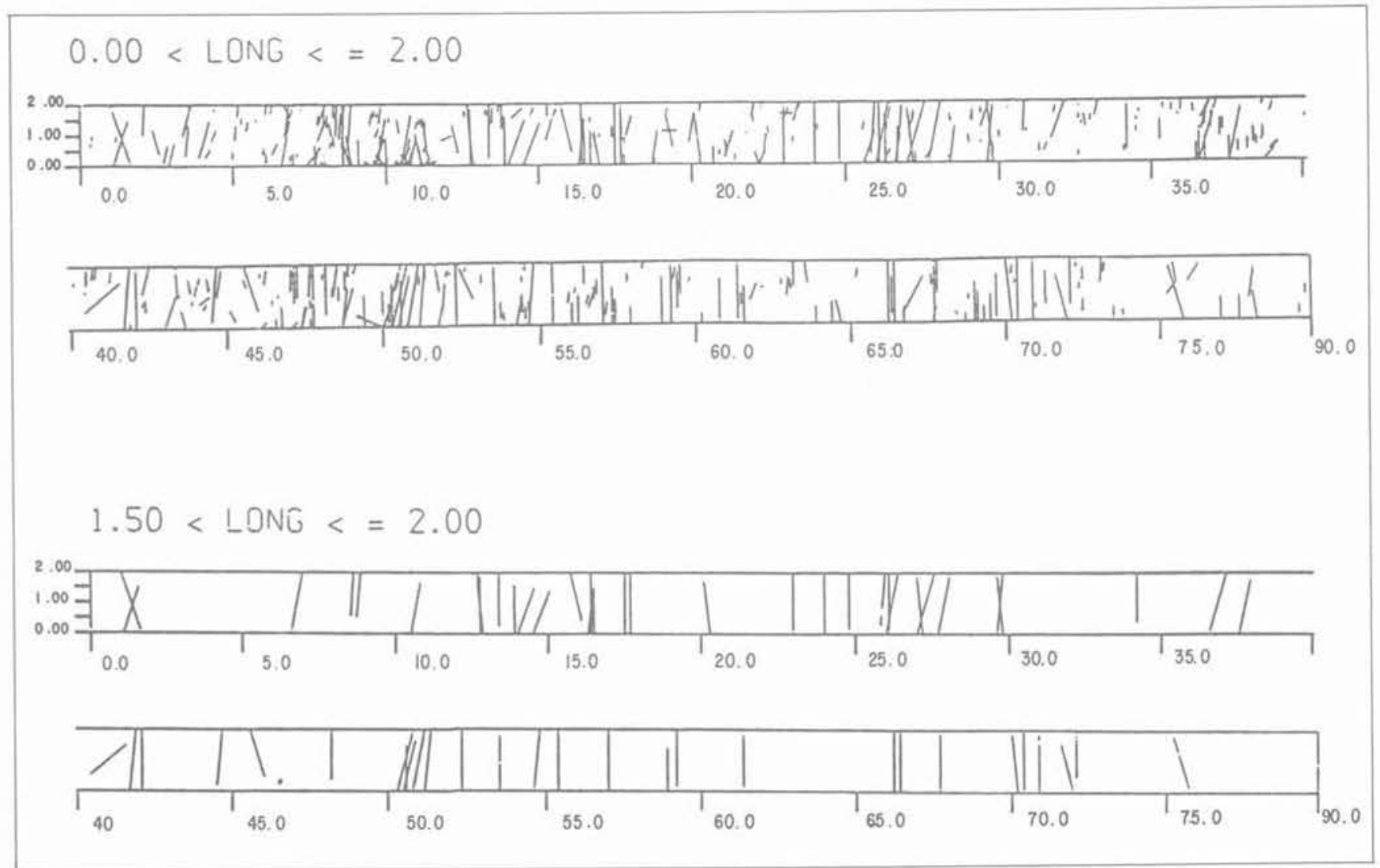

Fig. 2. - Organisation spatiale des fractures

d'après les relevés de H. MASSOUD - 1987.

Fig. 2. - Spatial organisation of fractures.

from measurements of H.MASSOUD - 1987.

ressérées autour d'un « germe », comme le montre la figure 2. De plus ces germes semblent se répartir aléatoirement dans la station, d'où la notion de germes poissoniens qui a été retenue. Toutefois, sur les variogrammes calculés aux grandes distances, une certaine variabilité est mise en évidence, qui est donc liée à une variabilité de la densité de germes. De cette constatation nait la démarche consistant à essayer d'améliorer le modèle de façon à rendre compte de cette structuration aux grandes distances, au moyen de l'étude des processus à densité régionalisée.

\section{Processus à densité régionalisée}

Ce dernier modèle, permet d'introduire une corrélation spatiale de la densité de fracturation entre supports voisins, c'est-à-dire une régionalisation de la densité $\varnothing_{\mathrm{g}}(\mathrm{x})$.

Il est possible de justifier ce modèle, qui semble correctement décrire la réalité au vu de la comparaison des variogrammes théoriques avec les variogrammes expérimentaux notamment, en considérant que l'hypothèse poissonienne rend compte du comportement des fractures au niveau local, la densité régionalisée étant associée à des facteurs tectoniques affectant l'ensemble des fractures, non considérées alors comme individus.

Le modèle de structure du phénomène " fracturation » étant relativement bien cerné, H.MASSOUD et J.P. CHILES avaient deux moyens à leur disposition en vue d'engendrer un champ de fractures. Pour simuler la distribution réelle des longueurs, ils pouvaient soit partir des relevés d'intersection des fractures sur les stations de mesure, soit partir d'un modèle a priori. En fait, l'application de méthodes et calculs de probabilité aux relevés de fracturation dont ils disposaient, leur a évité de choisir un modèle a priori. En effet, des calculs effectués sur des distributions marginales notamment permettent de remonter à la distribution des longueurs réelles des fractures.

Ainsi le principal programme de génération de champs de fractures, basé sur les conclusions des essais de différents modèles, s'organise-t-il de la façon suivante:

on définit d'abord une région de génération parallélé. pipédique contenant n.m.p. blocs eux aussi parallélépipédiques de dimensions n'.m'.p'. Les fractures sont modélisées par des disques d'épaisseur éventuellement variable. Le diamètre moyen des disques et l'écart-type sur ce diamètre sont ensuite donnés par l'utilisateur, 
ainsi que la dispersion selon les trois axes principaux du référentiel global. On se donne ensuite un plan vertical dans cette zone de génération, sur lequel on calcule le réseau formé par les intersections des fractures (disques) qui seront engendrées. Le nombre moyen des disques par salve est ensuite introduit par lutilisateur. Les germes sont engendrés par le programme selon une loi de Poisson dont on se fixe le paramètre. Les fractures sont alors engendrées de manière pseudo-aléatoire, de façon à ce que les spécifications données par l'utilisateur concernant les lois de distribution des diamètres, des espacements, des rayons, ou des germes, soient respectées.

Nous avons décrit, à partir d'un exemple, une méthode pour définir un modèle probabiliste de champ de fractures, puis le modèle lui-même, représentatif de la réalité telle qu'on peut la percevoir, grâce à l'emploi d'outils géostatistiques. Nous présentons, dans la partie suivante, le calcul des réseaux créés par des fractures, à partir de modélisations diverses; la modélisation par un schéma de salves poissoniennes à densité régionalisée devant pouvoir être une des modélisations prises en compte.

\section{GÉNÉRATION DU RÉSEAU DE FRACTURES}

Les programmes que nous allons présenter, initialement écrits dans le cadre des travaux de l'équipe de J. LONG au Lawrence Berkeley Laboratory, H.M. Peggy GILMOUR et al (1986), sont basés sur la modélisation suivante des fractures:

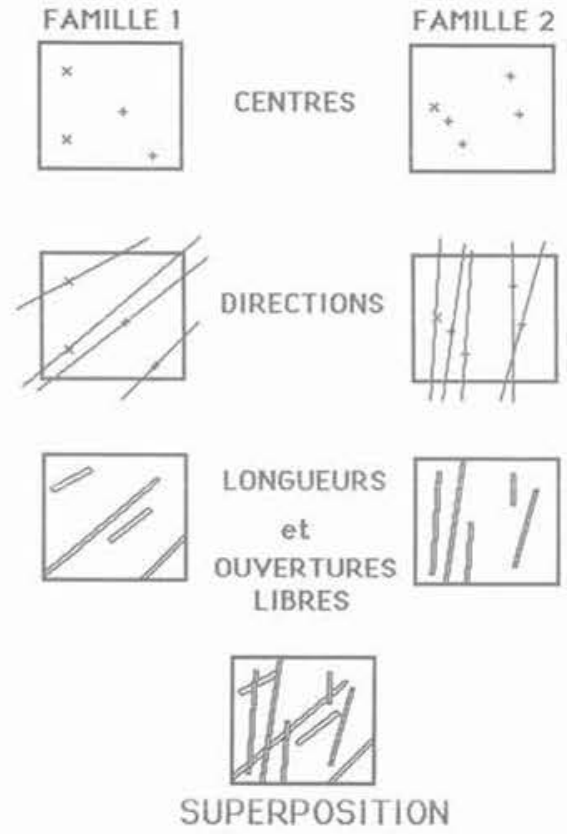

Fig. 3. - Génération à deux dimensions de fractures, famille par famille, avec superposition, d'après D. BILLAUX - 1986.

Fig. 3. - Generation, group by group, of fractures in two dimensions, and superposition. from D. BILLAUX - 1986 .
- en trois dimensions, chaque fracture, modélisée par un disque, est identifiée par sa position, son orientation, son rayon et son ouverture libre;

- en deux dimensions, elle est représentée par un segment de droite caractérisé par son centre, sa longueur, son orientation, et son ouverture libre, matérialisant l'intersection du cylindre plat tridimensionnel avec le plan d'étude.

Dans les deux cas, les fractures sont engendrées pseudo-aléatoirement, famille par famille, de façon indépendante, puis elles sont superposées, comme l'indique, pour le cas bidimensionnel, la figure 3 . L'originalité de notre approche, par rapport à d'autres, est de pouvoir introduire à ce niveau la structuration du phénomène mise en évidence, par les techniques géostatistiques: densités variables de germes, diversité des lois de distributions statistiques des rayons, écartements, et ouvertures des fractures simulées, sur la base de relevés expérimentaux.

Les distributions des longueurs de fractures et de leur ouverture libre peuvent être exponentielles, log-normales, ou gamma. Les distributions d'orientations peuvent être gaussiennes ou uniformes. Les fractures sont engendrées dans une région de génération parallélépipède rectangle, dans le cas bidimensionnel, dont les dimensions sont fixées par l'utilisateur. En trois dimensions, les mêmes lois de distribution peuvent s'appliquer aux populations de fractures. Celles-ci sont, dans ce cas, engendrées dans une région - toujours dite de \& génération * - de forme sphérique. On peut dans les deux cas bidimensionnel et tridimensionnel, entrer une à une les fractures, de façon déterministe.

Ce modèle de génération permet l'introduction simultanée de fractures positionnées de façon déterministe, et de façon pseudo-aléatoire. On peut également

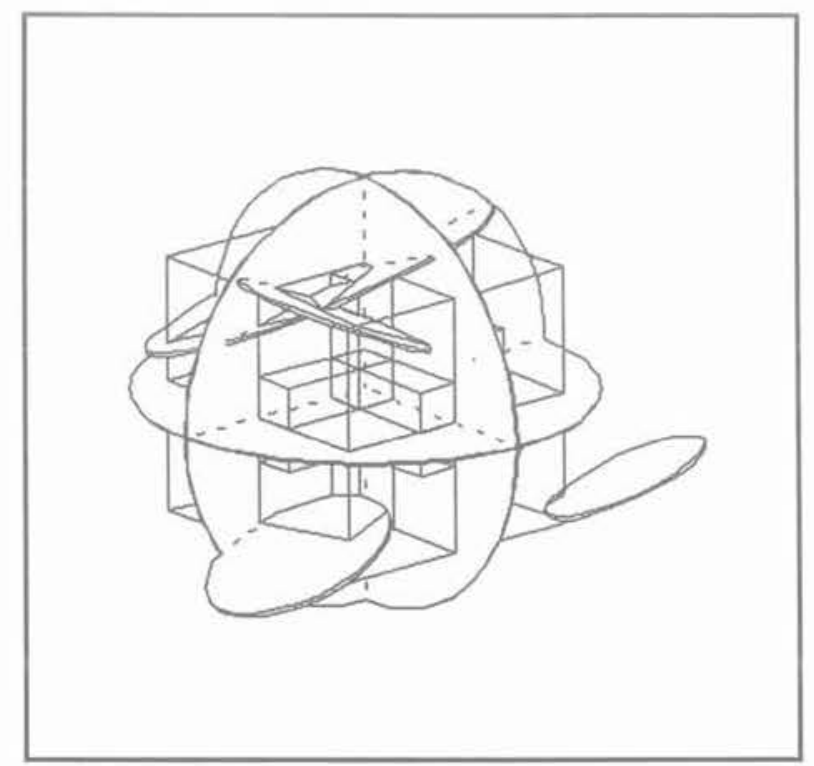

Fig. 4. - Exemple de génération de réseau de fractures, avec simultanément des fractures positionnées de façon déterministe, et engendrées pseudo-aléatoirement.

Fig. 4. - Example or fracture mesh generation including deterministically and statistically generated fractures. 
engendrer, de façon déterministe, des cavités à l'intérieur de la zone d'écoulement, de forme parallélépipé. diques. La figure précédente donne un exemple de telles réalisations (fig. 4). On remarquera trois cavités et trois fractures majeures perpendiculaires entre elles recoupant des fractures générées pseudo-aléatoirement, les paramètres statistiques de cette génération étant fixés par l'utilisateur.

Les fractures ayant été engendrées, on recherche alors les blocs de dimensions finies, découpés, ou définis par ces fractures qui se recoupent.

On étudie ensuite leur stabilité par la théorie des blocs clés. La reconnaissance de tous les blocs définis par des intersections de fractures fait encore partie des domaines dans lesquels notre recherche est, pour le moment, en cours (D. LIN et al., 1987).

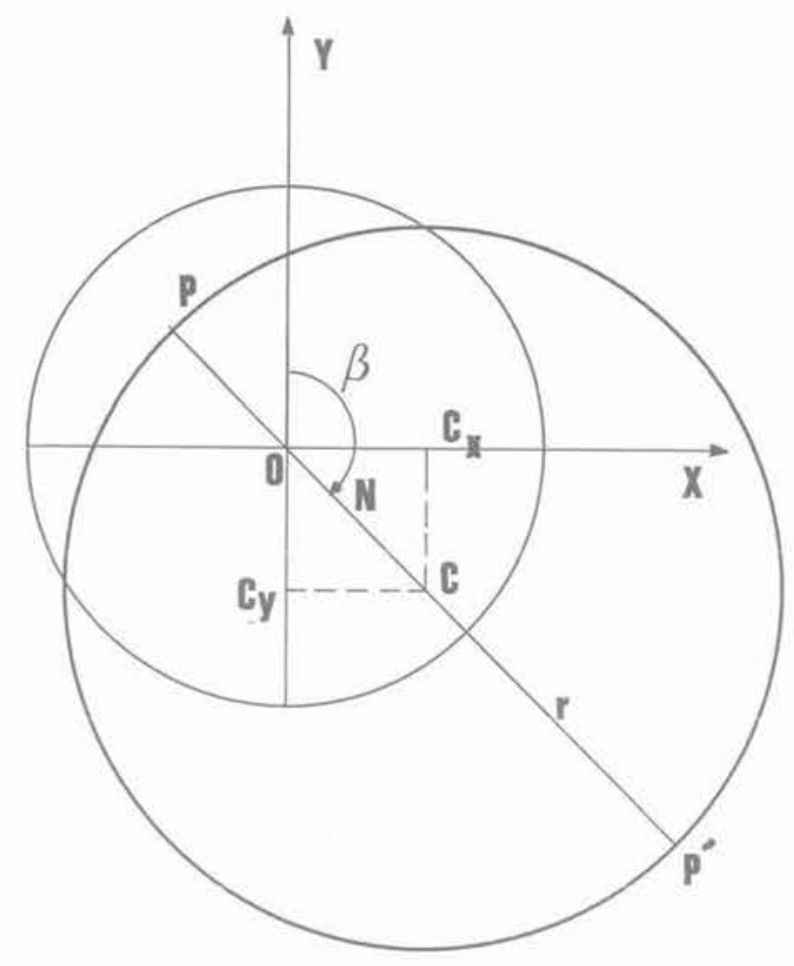

$$
\begin{aligned}
& \mathrm{ON}=\mathrm{R} \text { tang } \alpha / 2 \\
& \mathrm{OC}=\mathrm{R} \text { tang } \alpha \\
& \mathrm{r}=\mathrm{R} / \cos \alpha \\
& \mathrm{CX}=\mathrm{R} \operatorname{tang} \alpha \sin \beta \\
& \mathrm{Cy}=\mathrm{R} \operatorname{tang} \alpha \cos \beta
\end{aligned}
$$

Fig. 5. - Projection stéréographique d'un plan. Fig. 5. - Stereographic projection of a plane.

\section{NOTIONS SUR LA THÉORIE DU BLOC CLÉ, RESTREINTE A L'ÉTUDE DE LA STABILITÉ D'UN BLOC ISOLÉ}

Contrairement aux deux précédentes, cette dernière étape de la chaîne de traitement que nous proposons sera présentée comme un point plus bibliographique, basé essentiellement sur l'ouvrage de base de la théorie des blocs clés R.E. GOODMAN et G.H. SHI (1985). Nous renvoyons pour de plus amples développements à J.L YOW Jr. (1985), J.L. YOW Jr. et al (1987) W.J. BOYLE (1986).

Les principales hypothèses de base de la méthode sont:

a) les surfaces définissant les fractures sont parfaitement planes ;

b) l'étendue des plans de fractures est infinie, et il n'y a pas apparition de nouvelles fractures;

c) les blocs définis par les intersections des fractures sont considérés comme rigides;

d) les orientations, pendages et caractéristiques géométriques et mécaniques des joints sont donnés de façon déterministe.

En nous basant sur les propriétés géométriques de projections stéréographiques, nous pouvons présenter
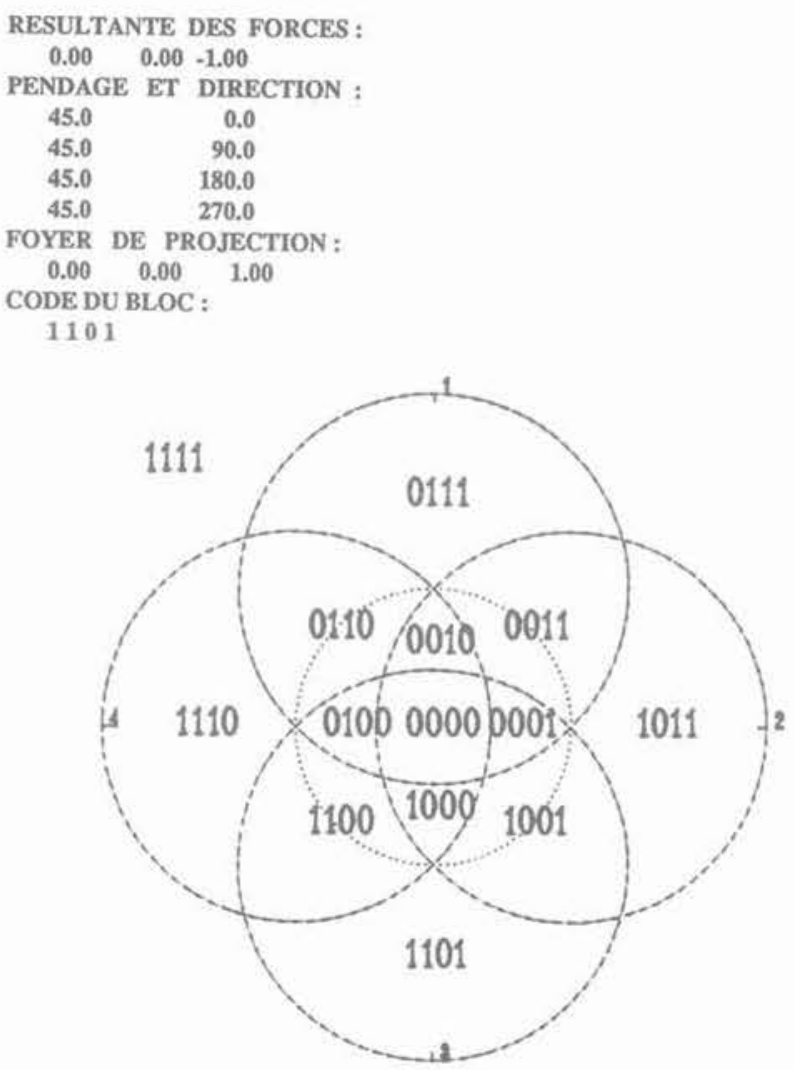

Fig. 6. - Exemple de projection d'intersections de demi-espaces, d'après des programmes originaux de R.E. GOODMAN et al.

Fig. 6. - Example of projection of intersections of half-spaces, from original programmes by R.E. GOODMAN et al. 
quelques aspects des résultats que l'on peut obtenir grâce aux programmes développés par l'équipe de R.E. GOODMAN, légèrement modifiés.

On notera que l'on peut associer à tout plan, de pendage $\alpha$ et de direction $\beta$ donnés, passant par le centre d'une sphère de référence choisie, sa projection sur le plan équatorial de cette sphère, qui est un cercle de caractéristique rappelées sur la figure 5 .

On peut remarquer que tout élément situé dans le demi-espace situé au dessus d'un plan P donné se projette à l'intérieur du cercle $\mathrm{C}$ qui est la projection de ce plan $\mathrm{P}$, dans le plan équatorial de la sphère de référence. Inversement, tout point situé au-dessous du plan $\mathrm{P}$ considéré est projeté à l'extérieur du cercle $\mathrm{C}$. Ainsi en associant un indice particulier à chacun des demi-espaces supérieur et inférieur, relativement à un plan donné, il est possible de définir pour un ensemble de $\mathrm{n}$ plans donnés une chaîne de n caractères, permettant de caractériser une intersection de $n$ demiespaces. On peut ainsi obtenir une partition du plan équatorial de la sphère de référence, et associer à chacune des régions définies par les intersections de demi-espaces supérieurs et inférieurs relatifs à des plans donnés, une chaîne de caractères donnée. La figure suivante, fig. 6 , donne un exemple de cette construction dans le cas de quatre plans.

A partir de cette étape, on peut choisir d'étudier la stabilité d'un bloc particulier, qui est défini par l'intersection de demi-espaces, relatifs à des plans donnés. On peut alors obtenir une représentation de ce bloc (fig. 7), en se fixant ses dimensions, ou obtenir, à titre d'exemple, la visualisation d'un bloc par rapport à une section de tunnel donnée, grâce aux programmes dé. veloppés par R.E. GOODMAN et al.

\section{SOMMET :}
5.62
2.02

PENDAGE ET DIRECTION :

45.0

0.0

45.0

45.0

180.0

$45.0 \quad 270.0$

DIRECTION DU TUNNEL :

$\begin{array}{lll}1.00 & 0.00 & 0.00\end{array}$

RESULTANTE DES FORCES :

$\begin{array}{llll}0.00 & 0.00 & -1.00\end{array}$

INTERVALLE ANGULAIRE :

\section{CODE DU BLOC :}

\section{1}

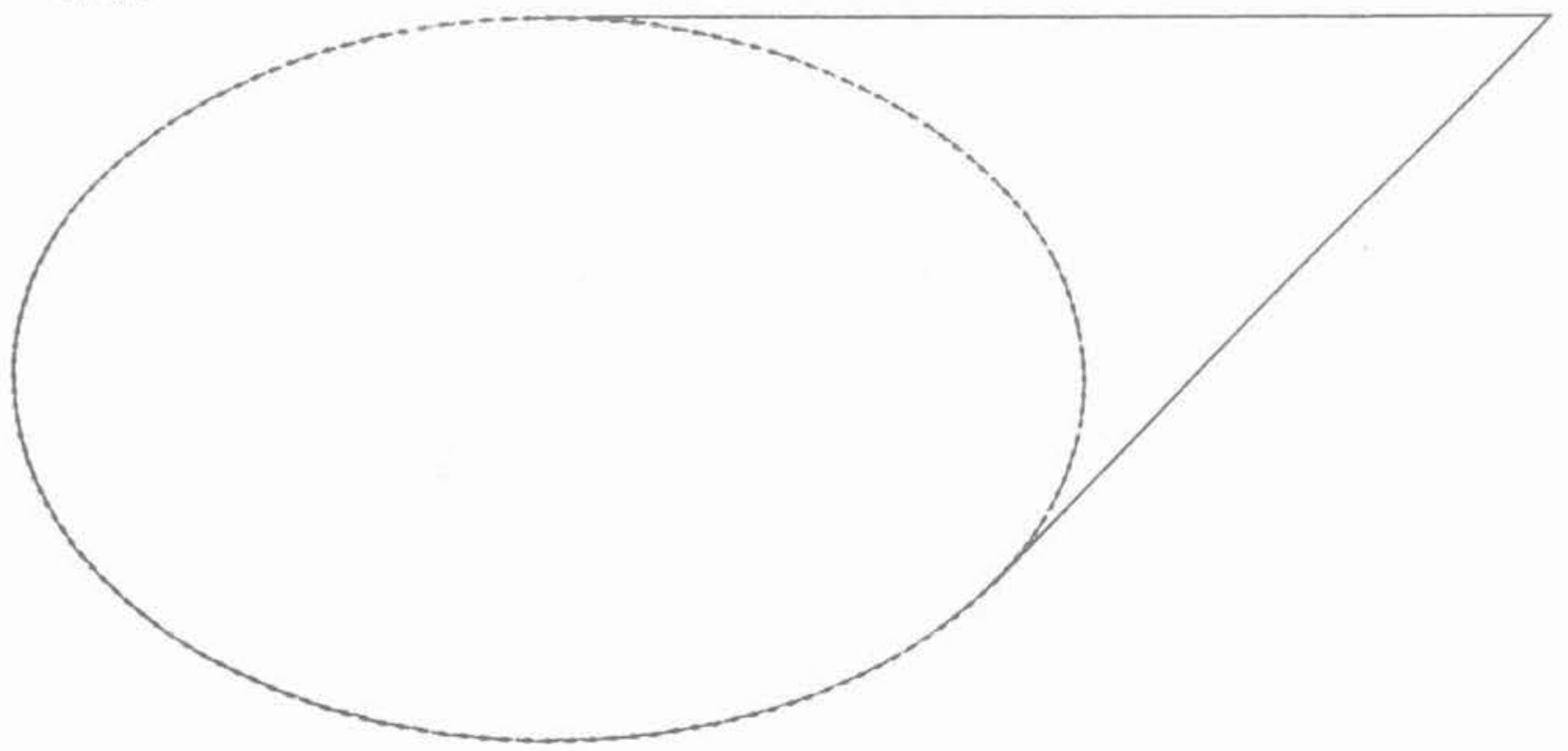

Fig. 7. - Exemple de résultats obtenus, d'après des programmes originaux de R.E. GOODMAN et al. Fig. 7. - Example of results, from original programmes by R.E. GOODMAN et al. 
Enfin, il est possible, à partir de la connaissance de la résultante des forces agissant sur un bloc donné, de déterminer si, pour un angle de frottement donné le long des faces du bloc, celui-ci est stable ou non, et dans ce dernier cas, connaître la direction du déplacement du bloc (glissement le long d'une des faces, le long de deux faces, soit encore parallèlement à la droite intersection des deux plans des faces, ou encore décollement du bloc). A titre d'exemple, la figure suivante, (fig. 8) donne pour la résultante des forces indiquée, $\mathrm{R}$, un mode de déplacement du bloc étudié le long de la face numéro 1 , tant que langle de frottement est inférieur à 55 degrés.

\section{REGIONS D ' EQUULIBRE PENDAGE DIRECTION COTE

$\begin{array}{lll}35.0 & 55.0 & 1 \\ 45.0 & 65.0 & 1 \\ 55.0 & 75.0 & 1 \\ 65.0 & 85.0 & 1\end{array}$

FOYER DE PROJECTION :

$\begin{array}{lll}0.00 & 0.00 & 1.00\end{array}$

INCREMENT SUR L'ANGLE : 15.00

FORCE RESULTANTE :

$\begin{array}{lll}0.00 & 0.00 & -1.00\end{array}$

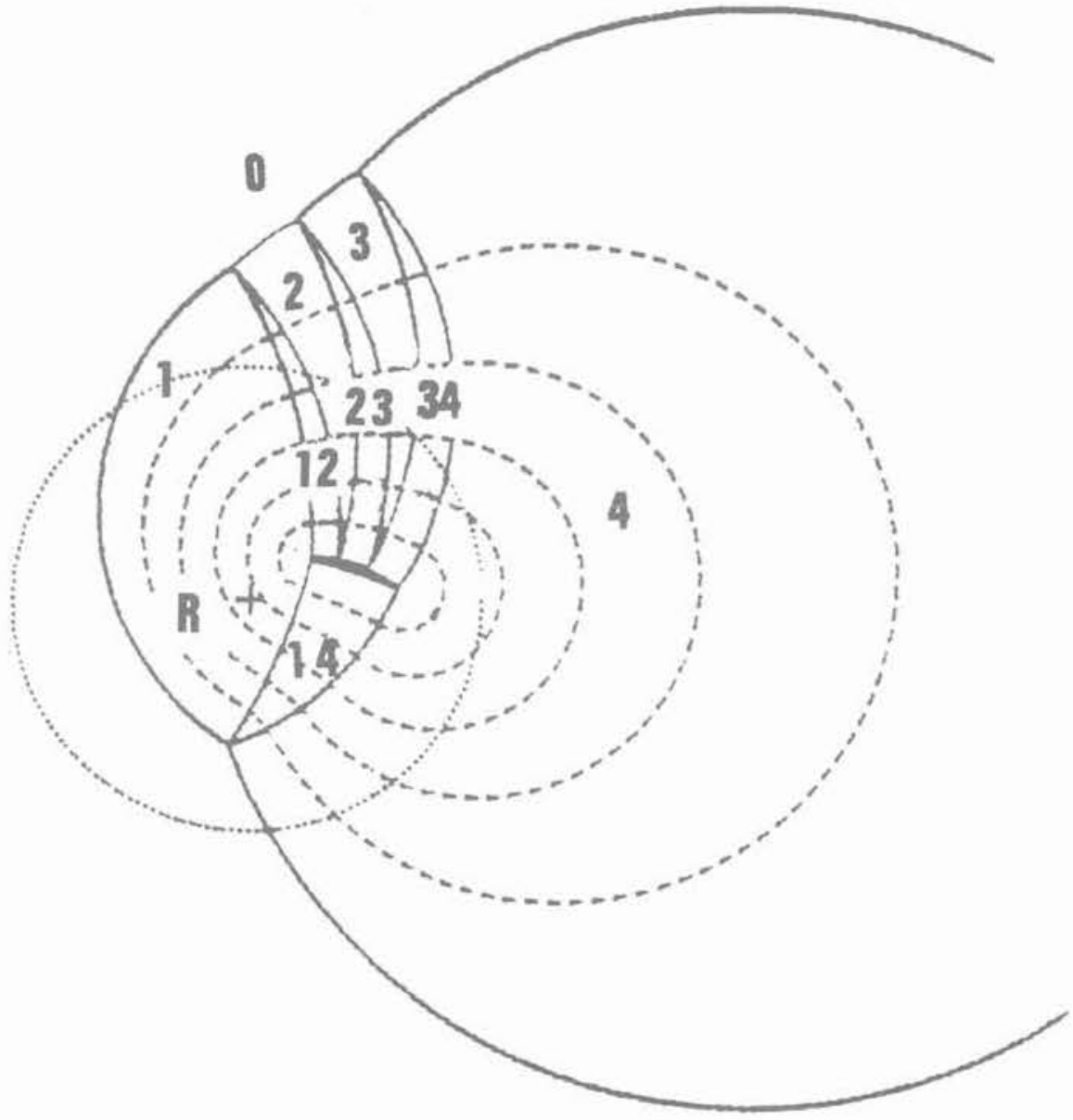

Fig. 8. - Exemples de détermination du mode de déplacement d'un bloc donné. D'après les programmes de R.E. GOODMAN et al.
Fig. 8. - Example of the determination of displacement mode of a given bloc. From original programmes by R. E. GOODMAN et al. 
Il est important de noter que les éléments de la théorie des blocs clés présentés ici s'intéressent à la stabilité d'un bloc isolé, et ne rendent pas compte des avantages de la méthode pour déterminer la meilleure orientation possible d'un ouvrage minier, compte tenu de la structure du massif. Cet aspect très important, peut toutefois être pris en compte, en redéfinissant l'orientation globale de l'ouvrage à réaliser, au niveau de la génération du réseau des fractures et des régions ou cavités qui l'intersectent, cavités qui peuvent représenter les travaux miniers à réaliser. Cette recherche d'optimisation est donc effectuée au niveau de la deuxième phase de la démarche concernant la génération du réseau, plus précisément au moment du positionnement des ouvrages à réaliser, et non au niveau de l'utilisation de la théorie des blocs-clés proprement dite. La détermination des différents blocs clés dans chaque cas permet, à partir de règles d'actions, d'orienter le choix du concepteur.

\section{CONCLUSION}

La chaîne de traitement des données structurales présentée ici doit permettre une prise de décision en meilleure connaissance de cause, tant au niveau de la conception que de l'exécution d'ouvrages au rocher. Par la possibilité qui est offerte d'engendrer simultanément des fractures de façon déterministe ou pseudo. aléatoire, l'ensemble du traitement permettra des applications dans divers cas de figure: stabilité de falaises, de travaux miniers, de talus routiers, conceptions de sites industriels souterrains...

A la différence de certaines approches, le massif rocheux fracturé n'est ici considéré ni comme un assem-

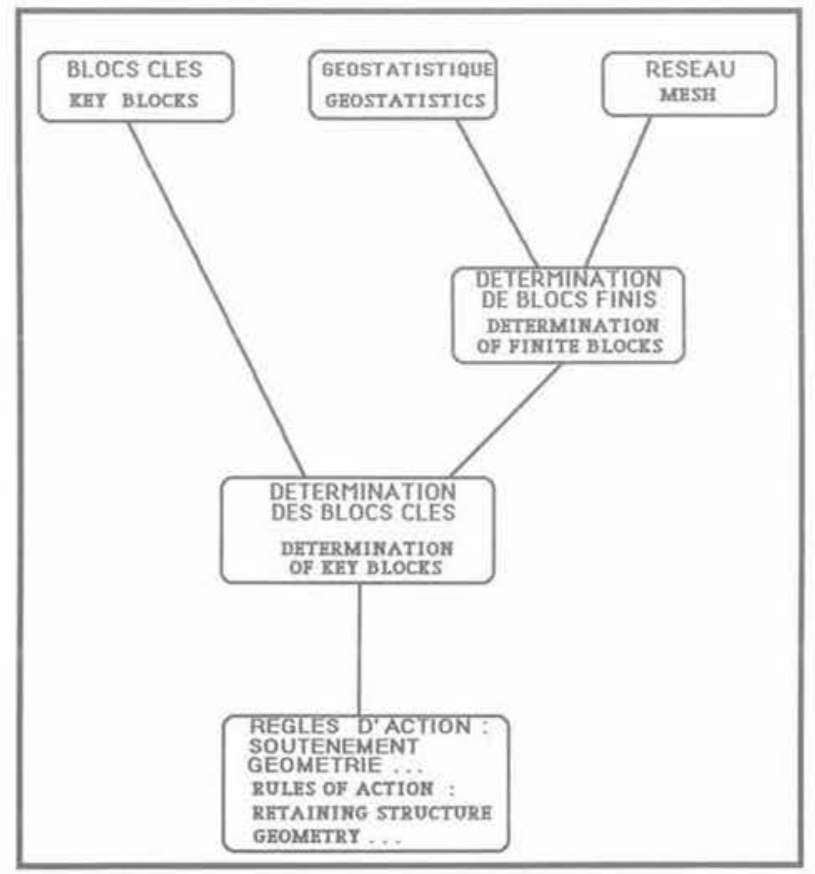

Fig. 9. - Schéma général du programme mis en place. Fig. 9. - General scope of the programme blage de blocs au sens strict, ni comme un milieu continu décomposé en sous-domaines ayant des propriétés égales à celles d'un matériau continu équivalent, mais plutôt comme une masse dans laquelle sont définis des blocs, délimités par des intersections de fractures de dimensions finies. La reconnaissance de tous les blocs finis, déterminés par ces intersections de fractures et de plans liés aux travaux entrepris dans le massif, présente encore des difficultés qui restent à surmonter. La figure 9 présente de façon très schématique l'ensemble du programme complet dont plusieurs parties fonctionnent déjà, mais de façons indépendantes.

Ce programme devrait bénéficier des résultats de travaux actuellement en cours à l'Institut des Matériaux et des Gisements Miniers, de l'Ecole des Mines d'Alès, portant sur la caractérisation et la prise en compte dans des modèles de calcul mécanique, de la rugosité des fractures rocheuses, et notamment de la dilatance. Par ailleurs, il utilise les versions améliorées des programmes de calcul de réseaux de fractures, en étroite collaboration avec le Département d'Ingéniérie Géotechnique du Bureau de Recherches Géologiques et Minières.

\section{REMERCIEMENTS}

La recherche dont les premiers résultats sont présentés dans cet article est effectuée dans le cadre du contrat $n^{\circ}$ MA $1 \mathrm{M}-0055$, entre la Commission des Communautés Européennes, et le B.R.G.M.

Y ont participé, en plus de l'auteur, Mademoiselle S. GENTIER et Messieurs J.P. CHILES et B. FEUGA pour le B.R.G.M., Messieurs S. BASTIDE, B. VAYSSADE, et $\mathrm{Ph}$. WEBER pour l'Ecole des Mines d'Alès.

\section{BIBLIOGRAPHIE}

1. BILLAUX D. (1986), Programmes de modélisation de champs de fractures en deux et trois dimensions, Document BRGM, 1986.

2. BOYLE W.J. (1986), The influence of initial stress on the movement of blocks around underground openings, Ph. D. dissertation, Univ. of California, Berkeley.

3. CHILES J.P. (1987), Three, dimensional geometric modeling of a fracture network, DOE/AECL'87 Conference \& Geostatistical sensitivity and uncertainty methods for ground-water flow and radionucleide transport modeling , SAN FRANCISCO Sept 15/17 1987.

4. CUNDALL P. (1980), UDEC, A generalised distinct element program for modeling jointed rock, Final technical report, U.S. ARMY, Contract $\mathrm{N}^{\circ}$. DAJA 37-79-C-0548, 1980.

5. CUNDALL P., HART R.D. (1985), Development of generalized 2-D and 3.D distinct element programs for modeling jointed rock, Final report, U.S. Army Corps of Engineers, Contract N ${ }^{\circ}$. DACA 39. 82-C-0015. 
6. FINE J., KORINI T. (1987), Une méthode d'analyse des risques de chutes de bloc dans les galeries creusées en terrains fissurés, 6 th Int. Cong. of ISRM, Montreal.

7. GILMOUR H.M. Peggy, BILLAUX D., S. LONG J.C. (1986), Models of calculation fluid flow in randomly generated three-dimensional networks of disc - shaped fractures, Rapport Lawrence Berkeley Laboratory LBL, 19515, Sept. 1986.

8. GOODMAN R.E., SHI G.H. (1985), Block theory and its application to rock engineering, Edt. Prentice-Hall, Inc. 338 pages.

9. HELIOT D., (1987), Generating a three dimensional jointed rock mass, Document interne CERCHAR.

10. LEMOS J., HART R., LORIG L.J. (1987), Dynamic analysis of discontinua using the distinct element method, 6 th lnt. Cong. of ISRM, Montreal.

11. LIN D., FAIRHURST C., STARFIELD A.M. (1987), Geometrical identification of three-dimensional rock systems using topological techniques, Int. J. Rock Mech. Min. Sci. \& Geomech. Abstr., Vol. $24 \mathrm{~N}^{\circ} 6$, pp. 331-338.

12. LORIG L.J., BRADY B.G.H., CUNDALL P.A. (1986), Hybrid distinct element - boundary element analysis of jointed rock, Int. J. Rock Mech. Min. Sci. \& Geomech. Abstr., Vol. $23 \mathrm{~N}^{\circ} 4$, pp. 303 312.

13. MASSOUD H., CHILES J.P. (1986), La modélisation de la petite fracturation par les techniques de la géostatistique, Rapport BRGM/Ecole des Mines d'Alès, Mai 1986.

14. MASSOUD H. (1987), Modélisation de la petite fracturation par les techniques de la géostatistique, thèse de Docteur-Ingénieur, Ecole des Mines de Paris, 1987.

15. VINCHES M. (1987), Approche des problèmes de stabilité des massifs rocheux fracturés, Rapport IRBAT, Juillet 1987.

16. VINCHES M., GERVAIS F., HETUIN E. (1988), Etude statistique et géostatistique de la fracturation de la mine des Bondons (France), à paraître, Document BRGM.

17. WARBURTON P.M. (1985), A computer program for reconstructing blocky rock geometry and analysing simple block stability, Computers and geosciences, Vol. $11 \mathrm{~N}^{\circ} 6$, pp. 707-712.

18. WARBURTON P.M. (1987), Implications of keystone action for rock bolt support and block theory, Int. J. Rock Mech. Min. Sci. \& Geomech. Abstr., Vol. 24, $\mathrm{N}^{\circ} 5$, pp. 283-290.

19. YOW Jr. J.L. (1985), Field investigation of keyblock stability, Ph. D. dissertation, Univ. of California, Berkeley.

20. YOW Jr J.L., GOODMAN R.E. (1987), A ground reaction curve based upon block theory, Rock Mech. and Rock Eng. 20, pp. 167-190.

21. YOW Jr J.L. (1987), Blind zones in the acquisition of discontinuity orientation data, Int. J. Rock Mech. Min. Sci. \& Geomech. Abstr., Vol. 24, N 5 , pp. $317-318$. 